\title{
Study on the relations between bloodstain age on the Cotton fabric with color and color difference
}

\author{
Baozhong Sun ${ }^{1, \star}$, Yichao $\mathrm{Cao}^{2,3}$, Kaijun $\mathrm{Ma}^{2}$, Xuejun Zhao ${ }^{2,3}$, Wei Zhang ${ }^{1}$, \\ Feng $\mathrm{Ji}^{1}$ \\ ${ }^{1}$ College of Textiles, Donghua University, Shanghai, China \\ ${ }^{2}$ Shanghai Key Laboratory of Crime Scene Evidence, Shanghai, China \\ ${ }^{3}$ Shanghai Research Institute of Criminal Science and Technology, Shanghai, China \\ *sunbz@dhu.edu.cn
}

Keywords: Cotton fabrics, Bloodstain age, Color difference.

\begin{abstract}
The accurate determination of time since death, or postmortem interval (PMI), can be critical in the investigation of suspicious deaths. Determine the bloodstain age on the fabric in crime scene is an important method for solving a case rapidly. This paper researches the relations between bloodstain age on the cotton fabric with color and color difference based on the color chromatic aberration method. This method provides a new perspective for determining the bloodstain age, also helps to solving a case quickly.
\end{abstract}

\section{Introduction}

Bloodstain, one of the most common and important traces at the scene of crime, usually remains on the clothing worn by human beings. If its age can be determined and analyzed, the time of death can be concluded, which will be of crucial significance for the quick detection of cases.

Currently, few methods have been found to determine the age of bloodstain, which is still a difficult problem in the fields of Forensic Medicine and Crime Scene Science. The method of measuring the chloride diffusion in the serum is one of the relatively commonly used methods, but it is affected by time and other factors and has great errors, thus it can only make a rough estimate. Anderson et al.[1] have used multiplexed, real-time RT-PCR (or qPCR) to determine the relative stability of different-sized segments of the same RNA species as well as differences in stability between two different RNAs' change over time in bloodstains. Phuvadol et al.[2] use smart phone cameras in combination with a truly low-cost illumination system as a tool to estimate the age of bloodstains. Gao et al. [3] use UV visible reflection spectrum to predict the age of bloodstains. Huang et al. [4] develop a DNA methylation assay for human age prediction in blood and bloodstain. Arany and Ohtani [5] estimate the age of bloodstain based on aspartic acid racemization rate. These researches are all helpful to determine the age of bloodstain.

This paper presents a new method, namely the color chromatic aberration method to study the relationship between the bloodstain age of the cotton fabric and the color chromatic aberration. The washed cotton fabric bloodstain samples after placed different time were measured by color chromatic aberration method.

\section{Materials}

Cotton fabrics, its specifications are as follows: plain weave, the linear density of warp yarn and weft yarn are both 20 tex. The warp density and weft density of the fabric are both $300 \mathrm{root} / 10 \mathrm{~cm}$. Warp and weft tightness are respectively $47.3 \%$ and $39.4 \%$. The total tightness is $68.1 \%$. The surface density is $136.5 \mathrm{~g} / \mathrm{m}^{2}$.

Blood: the fresh pig blood. The bloodstain cotton fabric sample is shown as Fig. 1. 


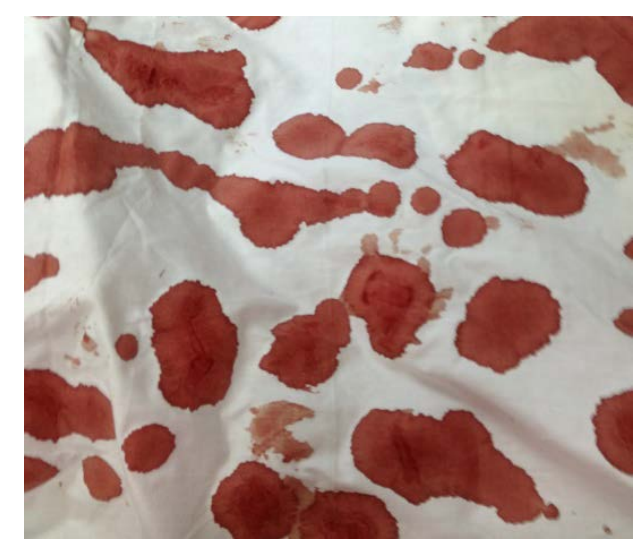

Fig. 1 Bloodstain cotton fabric sample

\section{Experimental}

Universal CR-310 tristimulus color meter is a small tristmulus color analyzer used in this test to gauge the reflected light of the sample's surface. It includes DP-301 data processor and measuring head. The color meter is used to test two different colors, choosing a color as the reference color, and then testing the sample color. The difference of two colors will be calculated and displayed on the screen. The computation formula is as follows:

$$
\Delta E^{*}=\left[\left(\mathrm{L}_{1}^{*}-\mathrm{L}_{0}^{*}\right)^{2}+\left(\mathrm{a}_{1}^{*}-\mathrm{a}_{0}^{*}\right)^{2}+\left(\mathrm{b}_{1}^{*}-\mathrm{b}_{0}^{*}\right)^{2}\right]^{\frac{1}{2}}
$$

Where $L_{0}^{*}, a_{0}^{*}$ and $b_{0}^{*}$ are the reference chroma value, $L_{1}^{*}, a_{1}^{*}$ and $b_{1}^{*}$ are the sample chroma value, $L^{*}$, $a^{*}$ and $b^{*}$ represent the brightness, red degree and yellow degree, respectively.

The blooded cotton fabric samples were placed different time at the room temperature. After finished the required time, the samples were washed for ten minutes. At last, the color difference of the sample was measured by the color meter.

\section{Results and discussion}

These samples placed at different time were washed and showed different colors, as shown in Fig. 2. It was found that the color of the sample without placed any time showed bright white. The bloodstain was almost disappeared. It indicated that the bloodstain fastness of cotton fabric was very low and easy to be washed at this condition. The color gradually deepening with the placed time increased. For the sample placed 2366h, the color was became dark brown, it means that the bloodstain was hard to be washed and the bloodstain fastness was very strong. Through qualitatively analyzing the blood color fastness of the above cotton fabric, we learn that the shorter placed time the blood stained fabrics, the smaller the fabric color fastness and the easier to wash. On the contrary, the longer placed time the blood stained fabrics, the harder to wash, forming much more color residues.

The washed cotton fabric bloodstain samples after placed different time were measured by color difference meter. The three indexes $\mathrm{L}, \mathrm{a}, \mathrm{b}$ about the color were obtained. The curves of different parameter values with the placing time of sample were plotted, as shown in Fig. 3, 4 and 5. 


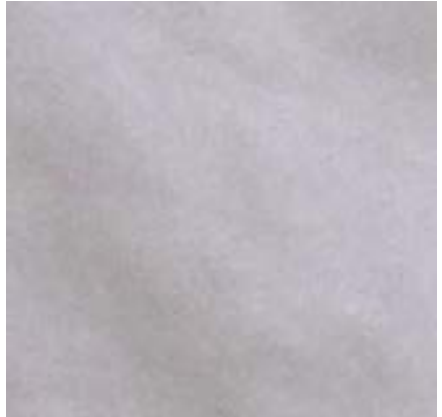

(1)0h

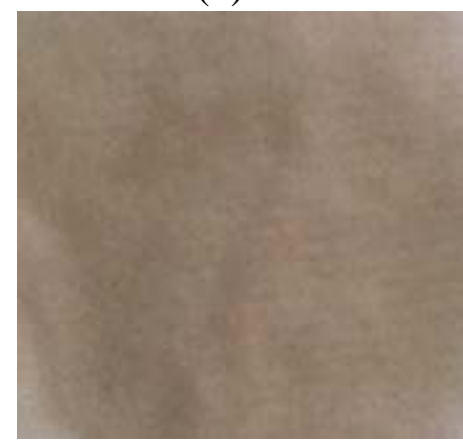

(4) $168 \mathrm{~h}$

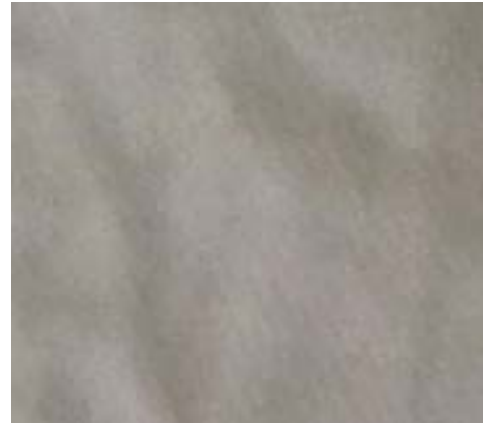

(2)14h

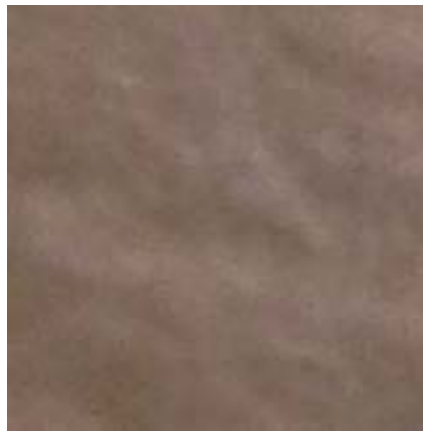

(5) $518 \mathrm{~h}$

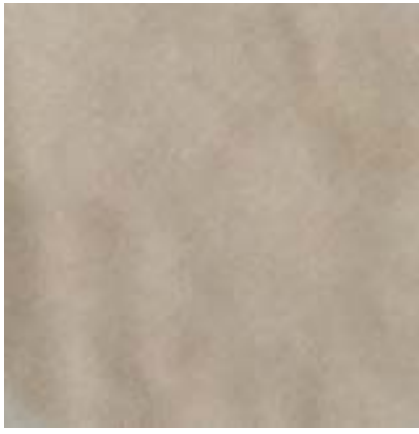

(3) $86 \mathrm{~h}$

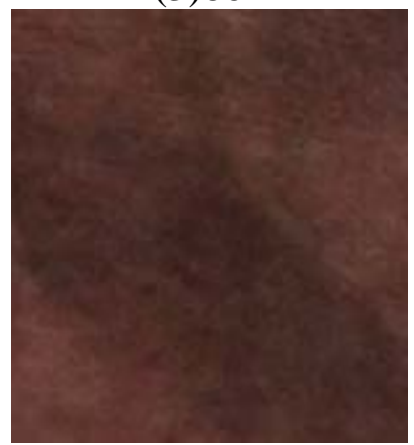

(6) $2366 \mathrm{~h}$

Fig. 2 The washed bloodstain samples after placing different time

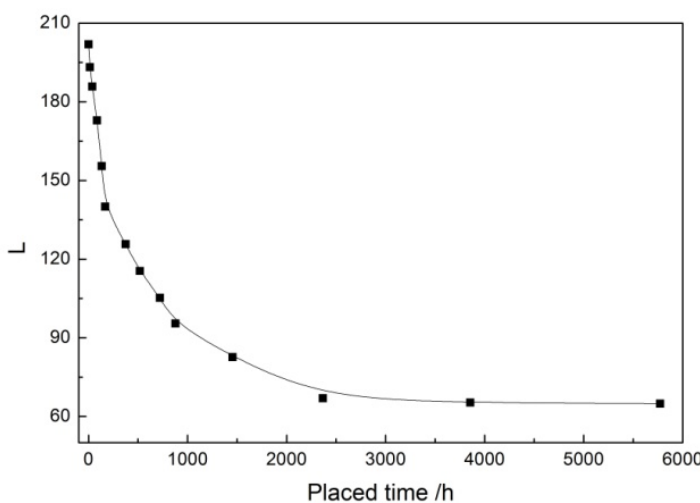

Fig. 3 The brightness L vs. placed time curve

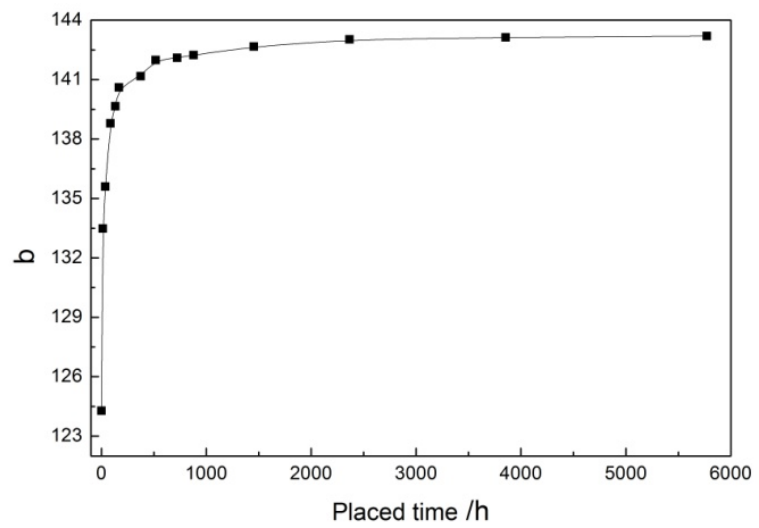

Fig. 5 The yellow degree b vs. placed time curve

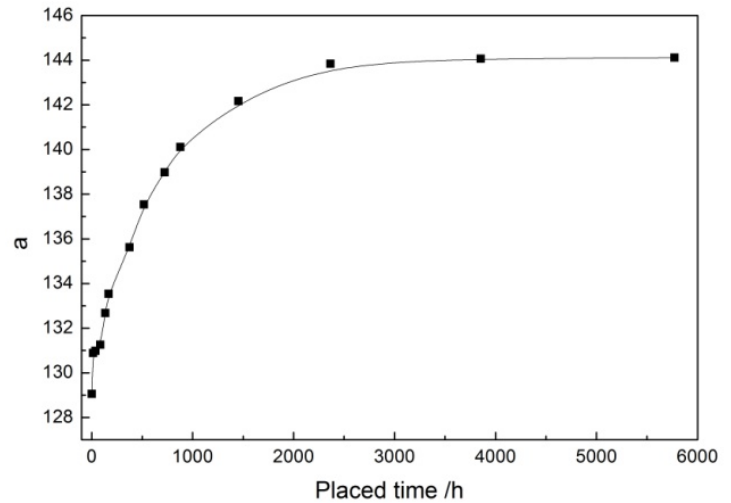

Fig. 4 The red degree a vs. placed time curve

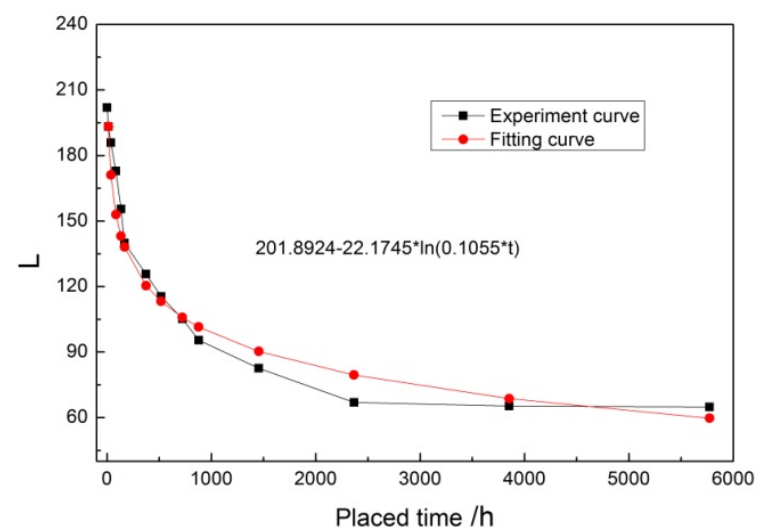
especially within the first $1000 \mathrm{~h}$. The L sharply reduces within $1000 \mathrm{~h}$. And then the L falls gradually until $3000 \mathrm{~h}$. The value keeps constant after $3000 \mathrm{~h}$ and there was no obvious change. It can be comparatively accurate determines the placed time of bloodstain samples by the L vs. placed time curves.

It can be found in Fig. 4 that the red degree index a increases with the increasing placed time, 
presenting an opposite change tendency compared with the brightness index L. The value increases dramatically with increasing placed time within $1000 \mathrm{~h}$, and then gradually increases until a stable platform after $3000 \mathrm{~h}$.

From Fig. 5, it can be seen that the yellow degree index b presents the same trend as the red degree index with the placed time. There are also three stages, but the yellow value increases rapidly within the first $50 \mathrm{~h}$, and then increases slowly until tends to be stable after $1500 \mathrm{~h}$.

As mentioned above, the three color indexes measured by the color different meter have a significant corresponding relation with the placed time of the bloodstain sample, especially within the first $1000 \mathrm{~h}$. It implies that this test has an effectiveness to predict the bloodstain age of cotton fabric within $3000 \mathrm{~h}$.

In order to forecast the placed time of bloodstain cotton fabrics, the brightness value $\mathrm{L}$ vs. the placed time curve was fitted, as shown in Fig. 6. The relation between brightness value and bloodstain age of samples is as shown in equation (2).

$$
L=201.8924-22.1745 * \ln (0.1055 * \mathrm{t})
$$

It is easy to know the placed time of bloodstain samples by testing the L. The red degree a vs. placed time curve and yellow degree b vs. placed time curve can be also obtain used the same method. The relationship between them no longer describe here.

\section{Conclusions}

The results described in this study show an effectiveness of determination of the bloodstain age of cotton fabric based on the color chromatic aberration method. The longer placed time the bloodstain cotton fabric, the harder to wash, and the deeper the color. The brightness degree index decreases with increasing the placed time. The red degree index increases with increasing the placed time, as well as the yellow degree index, but the changing extent is different. These three indexes are all relate to the placed time of bloodstain cotton fabric. It is easy to know the placed time of bloodstain samples by testing these three indexes. Determining the bloodstain age is helpful for solving a case quickly.

\section{Acknowledgements}

This work is supported by the Opening Project of Shanghai Key Laboratory of Crime Scene Evidence.

\section{References}

[1] Anderson, S. E., Hobbs, G. R. and Bishop, C. P.. Multivariate Analysis for Estimating the Age of a Bloodstain. Journal of Forensic Sciences, 2011(56): 186-193.

[2] Thanakiatkrai, P., A. Yaodam, and T. Kitpipit, Age estimation of bloodstains using smartphones and digital image analysis. Forensic Science International, 2013. 233(1-3): p. 288-297.

[3] Gao, Q.Y. and S.M. Gao, Authentication of Age of Bloodstains Using UV Visible Reflection Spectrum. Spectroscopy and Spectral Analysis, 2015. 35(8): p. 2221-2224.

[4] Huang, Y., Yan, J., Hou, J., Fu, X., Li, L., Hou, Y., Developing a DNA methylation assay for human age prediction in blood and bloodstain. Forensic Science International-Genetics, 2015. 17: p. 129-136.

[5] Arany, S. and S. Ohtani, Age estimation of bloodstains: A preliminary report based on aspartic acid racemization rate. Forensic Science International, 2011. 212(1-3): p. E36-E39. 